\title{
Growth During Tocilizumab Therapy for Polyarticular- course Juvenile Idiopathic Arthritis: 2-year Data from a Phase III Clinical Trial
}

\author{
Kamal N. Bharucha, Hermine I. Brunner, Inmaculada Calvo Penadés, Irina Nikishina, \\ Nadina Rubio-Pérez, Sheila Oliveira, Katarzyna Kobusinska, Heinrike Schmeling, \\ Flavio Sztajnbok, Frank Weller-Heinemann, Elena Zholobova, Francesco Zulian, Roger Allen, \\ Jeffrey Chaitow, James Frane, Chris Wells, Nicolino Ruperto, and Fabrizio De Benedetti, \\ for the Paediatric Rheumatology International Trials Organisation and the Pediatric \\ Rheumatology Collaborative Study Group
}

\begin{abstract}
Objective. Evaluate growth in patients with polyarticular-course juvenile idiopathic arthritis (pcJIA) treated with tocilizumab (TCZ) for up to 2 years in a phase III trial.

Methods. Patients with pcJIA lasting at least 6 months and inadequate response to methotrexate received open-label TCZ intravenously every 4 weeks (randomly assigned to 8 or $10 \mathrm{mg} / \mathrm{kg}$ if they weighed $<30 \mathrm{~kg}$; received $8 \mathrm{mg} / \mathrm{kg}$ if they weighed $\geq 30 \mathrm{~kg}$ ) for 16 weeks. Patients with JIAAmerican College of Rheumatology 30 response at Week 16 were randomly assigned to TCZ or placebo for 24 weeks, with an open-label extension through Week 104. Mean \pm SD height velocity (cm/yr) and World Health Organization (WHO) height SD score (SDS) were measured in patients receiving $\geq 1$ dose of TCZ who did not receive growth hormone and in patients whose baseline Tanner stage was $\leq 3$.

Results. The study included 187 of 188 patients (99.5\%) with mean WHO height SDS $-0.5 \pm 1.2$, which was unrelated to age or disease duration (Spearman rank correlations $r=0.08$ and $r=-0.12$, respectively). There were 123 patients at Tanner stage $\leq 3$ at baseline, among whom 103 completed the study with 2 years of height SDS data. Mean height SDS increased from baseline to year $2(+0.40$, $\mathrm{p}<0.0001)$. In 74 of 103 patients $(72 \%)$, height SDS was greater than at baseline, and mean height velocity was $6.7 \pm 2.0 \mathrm{~cm} /$ year.

Conclusion. Among patients with pcJIA at Tanner stage $\leq 3$ at baseline, $72 \%(74 / 103)$ had increased height SDS at the end of the study. (First Release July 1 2018; J Rheumatol 2018;45:1173-9; doi:10.3899/jrheum.170326)
\end{abstract}

Key Indexing Terms:

INTERLEUKINS BIOLOGICAL THERAPY GROWTH JUVENILE IDIOPATHIC ARTHRITIS

From Genentech, San Francisco; Consultant, Santa Monica, California, USA; Division of Rheumatology, Department of Pediatrics, University of Cincinnati, Cincinnati Children's Hospital Medical Center (CCHMC), Pediatric Rheumatology Collaborative Study Group (PRCSG)

Coordinating Center, Cincinnati, Ohio, USA; Paediatric Rheumatology Department, Hospital Universitario La Fe, Valencia, Spain; Pediatric Department, V.A. Nasonova Research Institute of Rheumatology; Department of Pediatric Rheumatology, Hospital of Childhood Diseases, JCA-UNIT, Sechenov Moscow Medical Academy, Moscow, Russia; Department of Pediatrics, Hospital Universitario Dr. J.E. González, Universidad Autónoma de Nuevo, Monterrey, Mexico; Universidade Federal do Rio de Janeiro, Instituto de Puericultura e Pediatria Martagao Gesteira (IPPMG); Hospital Universitario Pedro Ernesto, Nucleo de Estudos da Saúde do Adolescente, Universidade do Estado do Rio de Janeiro, Rio de Janeiro, Brazil; Wojewodzki Szpital Dzieciecy, Oddzial Pediatrii, Hematologii Onkologii i Reumatologii, Bydgoszcz, Poland; Department of Pediatrics, Alberta Children's Hospital and University of Calgary, Calgary, Alberta, Canada; Klinikum Bremen-Mitte, Professor Hess-Kinderklinik, Pädiatrische Intensivmedizin, Bremen, Germany; Rheumatology Unit, Department of Woman and Child Health, University of Padua, Padua; Istituto Giannina Gaslini, Pediatria II, Rheumatologia, Paediatric Rheumatology International Trials Organisation (PRINTO) Coordinating Centre, Genoa; Institute for Research and Health Care (IRCCS) Ospedale Pediatrico Bambino Gesù, Rome, Italy; Royal

\begin{abstract}
Children's Hospital, Pediatric Rheumatology, Melbourne, Victoria; Department of Rheumatology, Sydney Children's Hospital Network, Randwick and Westmead, Sydney, Australia; Roche Products Ltd., Welwyn Garden City, UK.

The study was funded by F. Hoffmann-La Roche Ltd. Writing and editorial services were provided by ApotheCom and funded by F. Hoffmann-La Roche Ltd. K.N. Bharucha is an employee of Genentech. H.I. Brunner reports funding from F. Hoffmann-La Roche Ltd. She is employed by CCHMC, which served as the North American PRCSG Coordinating Center. She is on the speaker's bureau of Genentech and serves as a consultant. I. Nikishina reports other funding from F. Hoffmann-La Roche Ltd. K. Kobusinska reports other funding from F. Hoffmann-La Roche Ltd. $H$. Schmeling reports grants from F. Hoffmann-La Roche Ltd., Janssen, Pfizer, and UCB Biosciences GmbH. J. Frane was a consultant for Genentech and has been employed as a consultant or an employee of Roche/Genentech. C. Wells is an employee of and owns shares in Roche. F. De Benedetti reports grants from Novartis, Roche, Pfizer, and Sanofi.

K.N. Bharucha, PhD, MD, Genentech; H.I. Brunner, MD, MSc, Division of Rheumatology, Department of Pediatrics, University of Cincinnati, CCHMC, PRCSG Coordinating Center; I. Calvo Penadés, $M D, P h D$, Paediatric Rheumatology Department, Hospital Universitario La Fe; I. Nikishina, MD, PhD, Pediatric Department, V.A. Nasonova Research Institute of Rheumatology; N. Rubio-Pérez, MD, Department of
\end{abstract}


Juvenile idiopathic arthritis (JIA) is a group of arthritides encompassing several forms of chronic arthritis with an onset before 16 years of age ${ }^{1}$. The effect of chronic arthritis on bone and joint development often results in impaired growth in children, ranging from a mild decrease in growth velocity to severely short stature. Patients with polyarticular-course JIA (pcJIA), and to a greater extent, systemic JIA (sJIA), experience impaired growth ${ }^{2,3,4,5,6}$. In patients with JIA, prolonged use of glucocorticoids has been associated with growth retardation ${ }^{7}$. Indeed, in a retrospective analysis, significant loss of height was positively correlated with duration of prednisone therapy, and even after the discontinuation of glucocorticoids, about $30 \%$ of patients with JIA did not achieve catch-up growth ${ }^{8}$. Given that significant growth retardation is also observed in steroid-naive children with sJIA and pcJIA, glucocorticoids are not solely responsible for impaired growth in patients with JIA ${ }^{9}$. A number of other factors contribute to growth retardation in JIA, including high disease activity and severity, disease duration, age at onset, malnutrition, genetic predisposition, demographics, and inflammation. Improvement in growth velocity and the attainment of normal height have been observed with improved disease control and reduced glucocorticoid dosing $5,10,11,12$.

The influence of proinflammatory cytokines on the pituitary-derived growth hormone insulin-like growth factor-1 (IGF-1) axis in growth retardation has been postulated ${ }^{4,13}$. Interleukin 6 (IL-6) appears to be the major proinflammatory cytokine involved in growth retardation in patients with JIA. A 50\% to $70 \%$ reduction in growth rate was observed in transgenic mice expressing high levels of circulating IL-6 compared with nontransgenic littermates. This growth defect, partially reversed by administration of an anti-IL-6 receptor antibody, was associated with low levels of IGF- $1^{14}$. Overexpression of IL-6 also led to

Pediatrics, Hospital Universitario Dr. J.E. González, Universidad Autónoma de Nuevo; S. Oliveira, MD, Universidade Federal do Rio de Janeiro, IPPMG; K. Kobusinska, MD, Wojewodzki Szpital Dzieciecy, Oddzial Pediatrii, Hematologii Onkologii i Reumatologii; H. Schmeling, MD, Department of Pediatrics, Alberta Children's Hospital and University of Calgary; F. Sztajnbok, MD, Hospital Universitario Pedro Ernesto, Nucleo de Estudos da Saúde do Adolescente, Universidade do Estado do Rio de Janeiro; F. Weller-Heinemann, MD, Klinikum Bremen-Mitte, Professor Hess-Kinderklinik, Pädiatrische Intensivmedizin; E. Zholobova, MD, Department of Pediatric Rheumatology, Hospital of Childhood Diseases, JCA-UNIT, Sechenov Moscow Medical Academy; F. Zulian, MD, Rheumatology Unit, Department of Woman and Child Health, University of Padua; R. Allen, MD, Royal Children's Hospital, Pediatric Rheumatology; J. Chaitow, MD, Department of Rheumatology, Sydney Children's Hospital Network; J. Frane, PhD, Consultant; C. Wells, Roche Products Ltd.; N. Ruperto, MD, MPH, Istituto Giannina Gaslini, Pediatria II, Rheumatologia, PRINTO Coordinating Centre; F. De Benedetti, MD, PhD, IRCCS Ospedale Pediatrico Bambino Gesù.

Address correspondence to Dr. F. De Benedetti, IRCCS Ospedale

Pediatrico Bambino Gesù, Piazza S. Onofrio 4, 00165 Rome, Italy. E-mail:fabrizio.debenedetti@opbg.net

Full Release Article. For details see Reprints and Permissions at jrheum.org Accepted for publication February 16, 2018. osteopenia with accelerated bone reabsorption, reduced bone formation, and defective ossification, indicating that IL-6 is an important mediator of bone homeostasis ${ }^{15}$. A statistically significant inverse correlation between growth velocity and serum IL-6 levels has been shown in patients with JIA ${ }^{3}$. IL-6 levels are markedly elevated in the serum and synovial fluid of patients with pcJIA and sJIA and have been correlated with disease activity and reduced growth ${ }^{3,16,17}$. Two pivotal phase III trials, TENDER ${ }^{18}$ and CHERISH ${ }^{19}$, have demonstrated the efficacy of the anti-IL- 6 receptor- $\alpha$ antibody tocilizumab (TCZ) in treating the signs and symptoms of sJIA and pcJIA. A posthoc analysis conducted in the TENDER study population demonstrated that TCZ promoted significant catch-up growth in patients with sJIA and improvement of laboratory markers associated with growth and bone homeostasis ${ }^{5}$. The aim of the present posthoc analysis was to evaluate growth rates in patients with pcJIA treated with TCZ in the 2-year CHERISH trial.

\section{MATERIALS AND METHODS}

Study design. The phase III CHERISH trial (ClinicalTrials.gov, NCT00988221) was conducted by members of the Paediatric Rheumatology International Trials Organisation (PRINTO) ${ }^{20}$ and the Pediatric Rheumatology Collaborative Study Group (PRCSG) to evaluate the efficacy and safety of TCZ in patients with pcJIA. Patients received intravenous TCZ every 4 weeks, dosed according to body weight $[\mathrm{BW} ; 8 \mathrm{mg} / \mathrm{kg}$ for $\mathrm{BW} \geq 30$ $\mathrm{kg}$ or randomly assigned (1:1) to receive $8 \mathrm{mg} / \mathrm{kg}$ or $10 \mathrm{mg} / \mathrm{kg}$ for $\mathrm{BW}<30$ $\mathrm{kg}]$. The trial consisted of 3 parts: a 16-week, open-label, lead-in period (Part 1); a 24-week, randomized, double-blind, placebo-controlled, withdrawal phase for patients who achieved at least a JIA-American College of Rheumatology 30 (ACR30) response during Part 1 (Part 2); and a 64-week, open-label extension (Part 3). Patients who experienced a JIA flare in Part 2 could escape to open-label TCZ. The study design and the full methodology of the CHERISH trial have been described ${ }^{19}$. The trial was conducted in accordance with the principles of the Declaration of Helsinki and good clinical practice guidelines and with local requirements. Informed consent from a parent or guardian and assent from the patient if applicable were obtained for all patients. The trial was approved by the institutions' Research Ethics Boards (the approval number for the principal trial site 165340 was 73/2009).

Patients. Eligible patients were 2 to 17 years of age and had pcJIA. The term pcJIA refers to rheumatoid factor-positive or-negative polyarticular JIA or extended oligoarticular JIA according to the International League of Associations for Rheumatology criteria ${ }^{21}$. Patients had to have disease for at least 6 months, inadequate response or intolerance to methotrexate (MTX), and at least 5 active joints at baseline. Stable doses of nonsteroidal antiinflammatory drugs, glucocorticoids, and MTX were permitted. Patients could not have been receiving oral glucocorticoids at the baseline visit, or if they were receiving oral glucocorticoids at that time, the dose had to have been stable $(\leq 0.2 \mathrm{mg} / \mathrm{kg} /$ day prednisone or $10 \mathrm{mg} /$ day, whichever was lower) for at least 4 weeks before and including the baseline visit. Glucocorticoid doses could be tapered in Part 3.

Assessments. Height was assessed at screening and baseline, at 2, 4, 8, 12 , 16, 20, and 24 weeks, and every 4 weeks thereafter through Week 104 Increases in growth over time were assessed using height SD scores (SDS) and height velocity $(\mathrm{cm} / \mathrm{yr})$. Height SDS was calculated as:

(observed height - mean value of the reference population) $\div$ $\mathrm{SD}$ of the reference population

The reference population was derived from age- and sex-matched World

Personal non-commercial use only. The Journal of Rheumatology Copyright $\odot$ (2018. All rights reserved 
Health Organization (WHO) norms ${ }^{22}$. Height SDS $>0$ indicated patients were above the mean height expected for their age and sex, whereas height SDS $<0$ indicated patients were below the mean height expected for their age and sex. Height velocity was calculated as:

(Year 1 or Year 2 height assessment - baseline height assessment) $\div$ (no. days between assessments $\div 365.25$ )

For this exploratory analysis, growth was assessed in a subset of patients who received $\geq 1$ dose of TCZ and did not receive growth hormone during the study. Because patients at Tanner stage $\leq 3$ represented children with the highest potential for growth, additional growth analyses for some assessments were performed in patients at Tanner stage $\leq 3$ at baseline for whom data were available at baseline, Year 1, and Year 2 (completer analysis, i.e., analysis of patients who completed the full $2 \mathrm{yrs}$ ). Height SDS at 2 years was also assessed in the subset of patients who had height SDS $<-2$ at baseline, representing patients with the greatest growth impairment.

Tanner stage was determined at selected visits during the study in accordance with published methods ${ }^{23}$. Given that genitalia growth and breast development may precede the growth of pubic hair, the genitalia score was used if pubic hair and genitalia scores were different in boys, and the breast development score was used if pubic hair and breast development scores were different in girls ${ }^{23}$. Tanner stage 1 indicates prepubertal development, and stages 2 to 5 indicate increasing sexual maturation.

Statistical analysis. Mean \pm SD was determined for height velocity and height SDS unless stated otherwise. The relationship between height SDS at baseline and age or disease duration was evaluated using Spearman rank correlation (r). Change in height SDS from baseline to Week 104 was assessed by 2 -sided paired $t$ test. $\mathrm{P}<0.05$ was considered statistically significant.

\section{RESULTS}

Baseline characteristics. In total, 188 patients were enrolled in the CHERISH study, and 187 (99.5\%) of those were included in our current analysis; 1 patient received growth hormone and was excluded from the analysis. During Part 2 of the study, 84 of 187 patients were randomly assigned to receive placebo and 3 of these patients discontinued before receiving a single dose; 39 of 81 patients (48.1\%) experienced a JIA flare and escaped to TCZ before the end of Part 2 (Week 40) ${ }^{19}$. Improvements in all JIA ACR core components with TCZ treatment were reported at Week $40^{19}$ and were maintained through Week $104^{24}$.

Baseline characteristics of the subset of patients $(n=187)$ who received TCZ and did not receive growth hormone (Table 1) were similar to those of the total study population ${ }^{19}$. Among 86 of 187 patients (46\%) who were receiving oral glucocorticoids at baseline, the mean dose at baseline was $0.13 \mathrm{mg} / \mathrm{kg} /$ day. In total, 123 of 187 patients (66\%) were at Tanner stage $\leq 3$ at baseline. Baseline height SDS was not related to age or disease duration (Spearman rank correlation $\mathrm{r}=0.08$ and $\mathrm{r}=-0.12$, respectively; Supplementary Figure $1 \mathrm{~A}$ and $1 \mathrm{~B}$, available with the online version of this article). Growth characteristics before and during TCZ treatment. At baseline, the mean height SDS was -0.5 among the patients who received TCZ and did not receive growth hormone; $30 \%$ $(56 / 187)$ of patients had a height SDS $<-1$, and 12\% (22/187) had a height SDS $<-2$ (Table 1). During 2 years of TCZ treatment, $55.9 \%(19 / 34)$ of male and $61.7 \%(74 / 120)$ of
Table 1. Baseline demographics and growth characteristics. Data are mean \pm SD unless otherwise noted.

\begin{tabular}{lc}
\hline Characteristics & Growth Analysis Subset ${ }^{\mathrm{a}}, \mathrm{n}=187$ \\
\hline Female, $\mathrm{n}(\%)$ & $143(76)$ \\
Age, yrs & $11.0 \pm 4.0$ \\
Tanner stage $\leq 3, \mathrm{n}(\%)$ & $123(66)$ \\
Duration since pcJIA diagnosis, yrs & $4.2 \pm 3.7$ \\
Height SDS (WHO) & $-0.5 \pm 1.2$ \\
$<-1, \mathrm{n}(\%)$ & $56(30)$ \\
$<-2, \mathrm{n}(\%)$ & $22(12)$ \\
BMI SDS (WHO) & $0.1 \pm 0.1$
\end{tabular}

a Patients who received $\geq 1$ dose of TCZ and did not receive growth hormone. BMI: body mass index; pcJIA: polyarticular-course juvenile idiopathic arthritis; SDS: standard deviation score; TCZ: tocilizumab; WHO: World Health Organization.

female patients experienced height velocities greater than the mean height velocity for their respective ages. There was no evident difference in 2-year height velocity in the 81 patients who received placebo for a mean duration of about 18 weeks in Part 2 of the trial (Supplementary Table 1, available with the online version of this article) compared with those who received TCZ continuously (Figure 1A and 1B). Overall, the mean height SDS at weeks 52 and 104 were $-0.3 \pm 1.1$ and $-0.2 \pm 1.1$, respectively. The mean height SDS increased by +0.3 from baseline to Year 2 in patients who had height SDS data at both timepoints $(n=154)$.

Among patients at baseline Tanner stage $\leq 3(n=123)$, 103 had data to Week 104 of the study. In these patients, mean height SDS increased significantly from baseline at $-0.6 \pm 1.1$ to Year 1 at $-0.4 \pm 1.2$ and to Year 2 at $-0.2 \pm 1.1$ ( $\mathrm{p}<0.001$ at both timepoints; Figure 2A and 2B; Supplementary Table 2, available with the online version of this article). Thus, mean height SDS increased by 0.4 from baseline to Year 2. At Week 104, height SDS increased compared with baseline in 74 of 103 of these patients $(72 \%)$; the mean height velocity in these 103 patients was $6.7 \pm 2.0$ $\mathrm{cm} /$ year during the 2 -year period. These results in patients at baseline Tanner stage $\leq 3$ were consistent with observations for the entire growth population (Supplementary Figure 2 and Supplementary Table 2). Among 11 patients who were at Tanner stage $\leq 3$ and had height SDS $<-2$ at baseline, height SDS was improved in 8 patients at Year $2(\mathrm{p}=0.012)$, with a mean increase in height SDS of 0.57 (Table 2).

\section{DISCUSSION}

At baseline, height SDS was below the 50th percentile in a large proportion of patients with pcJIA enrolled in the CHERISH trial. Overall, growth profiles improved in patients treated with TCZ through the 2 years of the study, with a mean increase in height SDS of +0.3 from baseline to Year 2 and +0.4 in patients at Tanner stage $\leq 3$ at baseline. Most $(72 \%)$ of the patients at Tanner stage $\leq 3$ at baseline had increased height SDS at the end of the study (Yr 2), and most

Personal non-commercial use only. The Journal of Rheumatology Copyright (c) 2018. All rights reserved. 


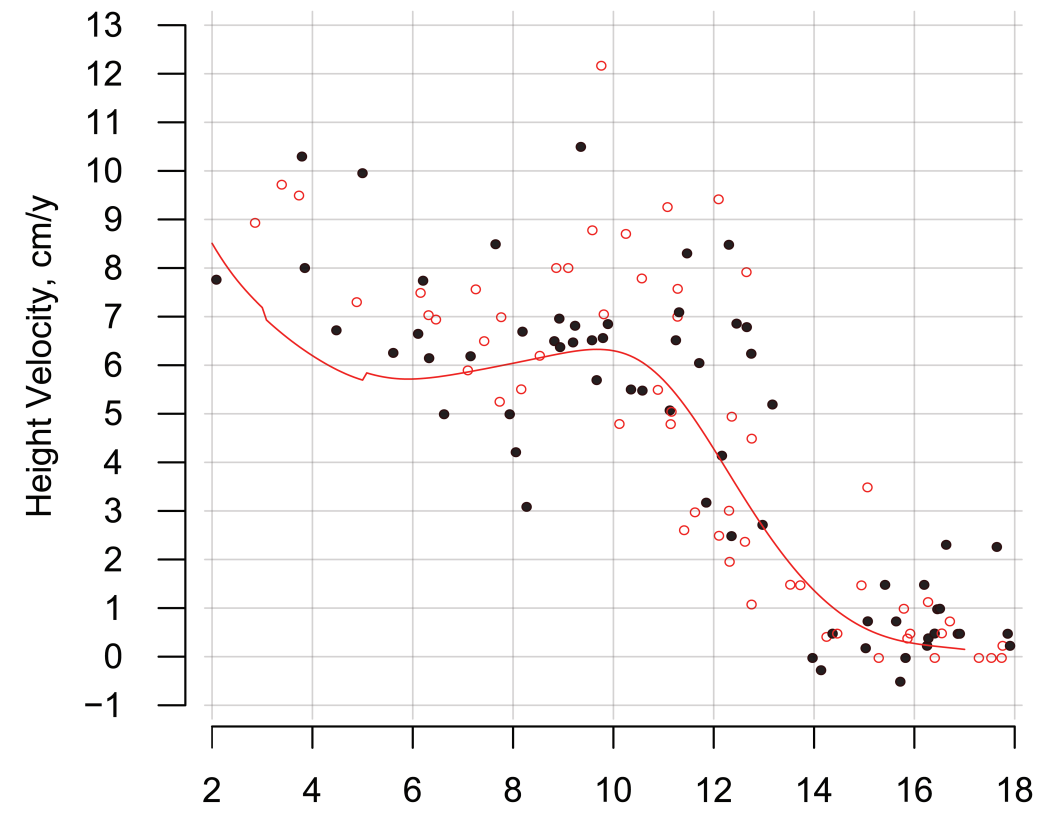

Age, years

B

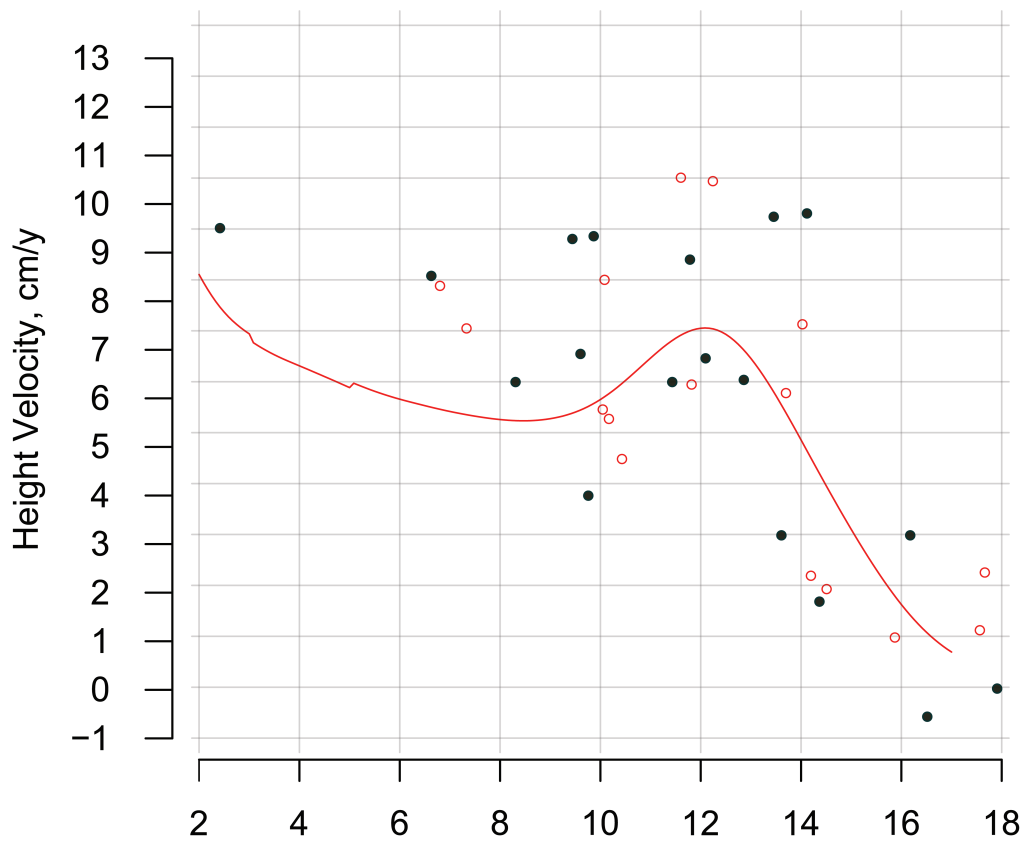

Age, years
Figure 1. Height velocity during 2 years versus baseline age in (A) girls $(\mathrm{n}=120)$ and $(\mathrm{B})$ boys $(\mathrm{n}=34)$ according to randomization to placebo or TCZ treatment during Part 2. Actual height velocities are a function of age and pubertal status. Solid red curves represent the expected 2-year annualized height velocities based on World Health Organization norms. Eighty-four patients were randomly assigned to receive placebo during the Part 2 withdrawal phase; about $50 \%$ of those patients escaped to TCZ before Week 40. Red open circles represent patients who were randomly assigned to the TCZ treatment group during Part 2 (61 girls, 16 boys) and who received TCZ continuously for 2 years. Black dots represent patients who were randomly assigned to the placebo group during Part 2 (59 girls, 18 boys). TCZ: tocilizumab. 
A

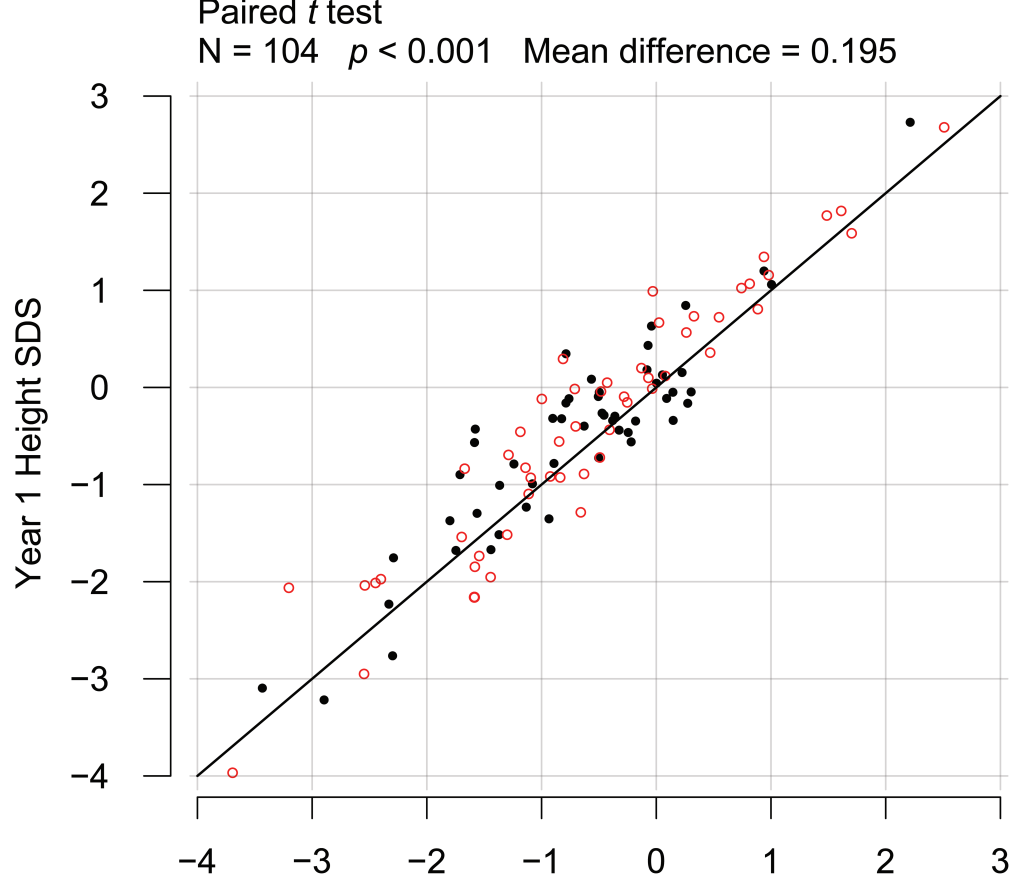

Baseline Height SDS

B

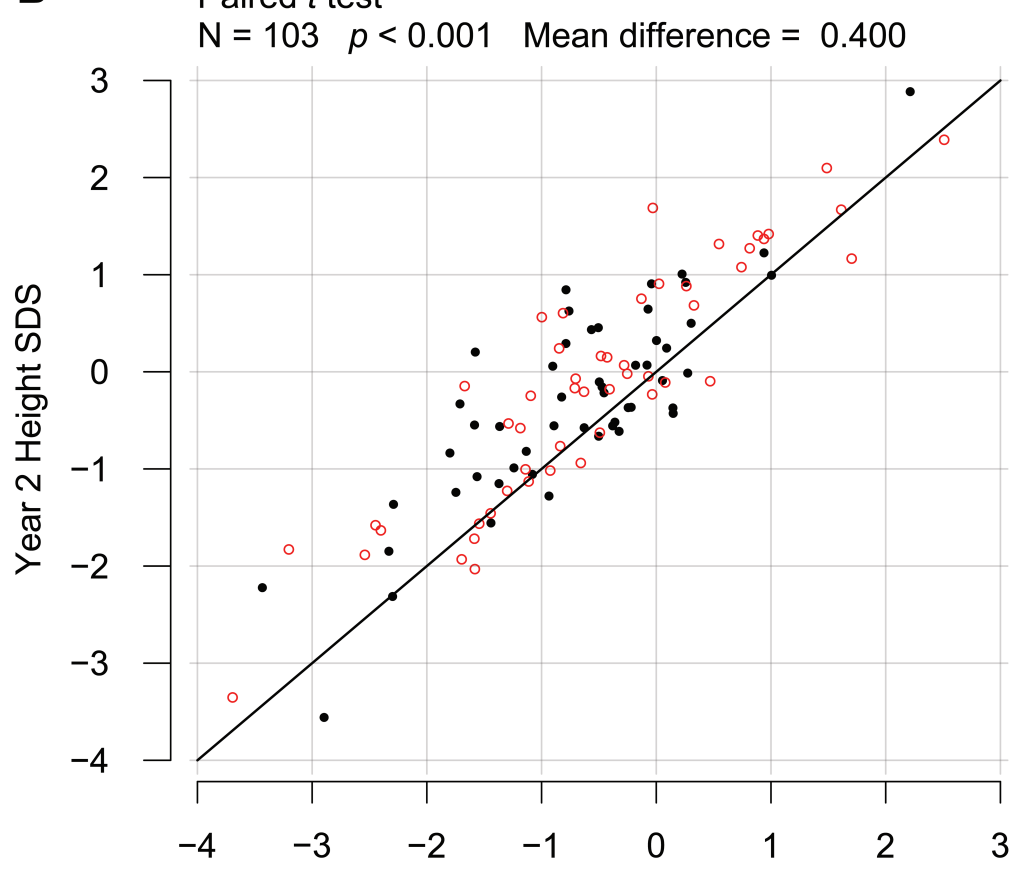

Baseline Height SDS
Figure 2. Baseline height SDS versus height SDS at (A) Year 1 and (B) Year 2 in patients at baseline Tanner stage $\leq 3(\mathrm{n}=103)$. Eighty-four patients were randomly assigned to receive placebo treatment during the Part 2 withdrawal phase; about $50 \%$ of those patients escaped to TCZ before Week 40. Black diagonal line represents no change in height SDS. Black dots represent patients randomly assigned to placebo in Part 2. Red open circles represent patients who received TCZ continuously. SDS: standard deviation score; TCZ: tocilizumab. 
Table 2. Height SDS for patients at baseline Tanner stage $\leq 3$ and baseline height SDS $<-2(\mathrm{n}=11)^{\mathrm{a}}$

\begin{tabular}{lccc}
\hline Baseline Age, Yrs/sex & Baseline Height SDS & Year 1 Height SDS & Year 2 Height SDS \\
\hline 8/female & -2.879 & -3.203 & -3.545 \\
11/female & -2.314 & -2.218 & -1.833 \\
10/female & -2.530 & -2.936 & $\mathrm{NA}$ \\
14/male & -3.417 & -3.082 & -2.209 \\
10/female & -2.524 & -2.024 & -1.870 \\
12/female & -3.186 & -2.049 & -1.815 \\
10/female & -2.430 & -1.999 & -1.566 \\
12/male & -2.282 & -2.749 & -2.299 \\
7/female & -2.384 & -1.960 & -1.619 \\
12/female & -2.273 & -1.740 & -1.350 \\
8/female & -3.676 & -3.954 & -3.339 \\
Mean height SDS $\pm \mathrm{SD}$ & $-2.72 \pm 0.497$ & $-2.54 \pm 0.694$ & $-2.14 \pm 0.741$ \\
\hline
\end{tabular}

a Eleven patients had baseline and Year 1 data, and 10 patients had baseline and Year 2 data. NA: not available; SDS: standard deviation score.

male and female patients also experienced increased height velocities. In a similar growth analysis of patients with sJIA in the TENDER trial, catch-up growth was observed over 2 years of treatment with $\mathrm{TCZ}^{5}$. Patients in the TENDER trial had a lower height SDS at baseline (-2.2), which was indicative of more severe growth impairment in patients with sJIA $^{2}$ and was likely attributed to higher inflammatory burden, greater need for longterm glucocorticoid therapy, or both. In the CHERISH growth analysis, 8 of the 11 patients who had height SDS $<-2.0$ at baseline had markedly improved height SDS at Year 2, suggesting that patients with pcJIA who had the most severe growth impairment experienced growth improvement. In the TENDER trial, the mean increase in height SDS from baseline to Year 2 was +0.3 in patients at Tanner stage $\leq 4$ at Year 2, possibly indicative of the lower baseline height SDS and the greater potential for catch-up growth. Nevertheless, in both studies, the mean height SDS was significantly increased over 2 years of TCZ treatment.

In the TENDER trial, multiple regression analysis revealed a relationship between height velocity during Year 1 and age at baseline and average glucocorticoid dose during Year 1 in patients with sJIA. The relationship with age was attributed to the higher growth potential in younger children, suggesting that early intervention is required to maximize therapeutic benefit on growth ${ }^{5}$. The correlation with glucocorticoid dose would be expected given the effect of glucocorticoids on growth impairment ${ }^{5,25}$. Multiple regression analysis was investigated for the present CHERISH study, but the resultant total $\mathrm{R}^{2}$ value $(0.24)$ was not considered large enough to be clinically relevant; this might have resulted from the normal growth trajectories observed for patients with pcJIA treated with TCZ in CHERISH compared with the catch-up growth observed in TENDER for patients with sJIA treated with TCZ who had overall short stature at baseline ${ }^{5}$. In addition, 1 important difference was that fewer patients were receiving glucocorticoids at baseline in CHERISH (46\%) than in TENDER (90\%). A limitation of our study might be the exclusion of patients receiving high doses of glucocorticoids in CHERISH, which could have affected the overall baseline height SDS of the study population because patients receiving higher doses of glucocorticoids would likely have experienced more severe growth impairment as a result of this treatment. Therefore, growth patterns during the study might have been influenced by glucocorticoid dosing at baseline and glucocorticoid tapering during the study as well as by the effect of TCZ treatment. The exclusion of patients who did not achieve JIA ACR30 response after Week 16 in CHERISH might have led to a selection bias for patients who had greater growth potential while receiving TCZ treatment.

A further limitation compared with the TENDER trial is the lack of analysis of laboratory variables, including IGF-1 levels and markers of bone turnover. In the TENDER analysis, TCZ treatment was associated with normalization of IGF-1 levels, and baseline levels of osteocalcin (a marker of bone formation) and C-telopeptide of type I collagen (a marker of bone resorption) were related to levels of C-reactive protein (CRP), a marker of inflammation. It remains to be determined whether TCZ has an effect on markers of growth or bone turnover in patients with pcJIA; however, given the reduction in CRP in $\mathrm{CHERISH}^{19}$, it is reasonable to expect that there might be a similar relationship with bone turnover and IGF-1.

Most patients with pcJIA in the CHERISH study at baseline Tanner stage $\leq 3$ experienced increased height SDS at the end of Year 2, consistent with improved growth profiles previously reported for patients with sJIA treated with TCZ. Overall, patients with pcJIA experienced adequate or improved growth over 2 years of treatment with TCZ, particularly those whose growth impairment at baseline was greatest.

Personal non-commercial use only. The Journal of Rheumatology Copyright $\odot$ (2018. All rights reserved 


\section{ONLINE SUPPLEMENT}

Supplementary material accompanies the online version of this article.

\section{REFERENCES}

1. Ravelli A, Martini A. Juvenile idiopathic arthritis. Lancet 2007;369:767-78.

2. Liem JJ, Rosenberg AM. Growth patterns in juvenile rheumatoid arthritis. Clin Exp Rheumatol 2003;21:663-8.

3. Souza LS, Machado SH, Brenol CV, Brenol JC, Xavier RM. Growth velocity and interleukin 6 concentrations in juvenile idiopathic arthritis. J Rheumatol 2008;35:2265-71.

4. Bechtold S, Simon D. Growth abnormalities in children and adolescents with juvenile idiopathic arthritis. Rheumatol Int 2014;34:1483-8.

5. De Benedetti F, Brunner H, Ruperto N, Schneider R, Xavier R, Allen R, et al; Paediatric Rheumatology International Trials Organisation and the Pediatric Rheumatology Collaborative Study Group. Catch-up growth during tocilizumab therapy for systemic juvenile idiopathic arthritis: results from a phase III trial. Arthritis Rheumatol 2015;67:840-8.

6. Kearsley-Fleet L, Hyrich KL, Davies R, Lunt M, Southwood TR; British Society for Paediatric and Adolescent Rheumatology Etanercept Cohort Study. Growth in children and adolescents with juvenile idiopathic arthritis over 2 years of treatment with etanercept: results from the British Society for Paediatric and Adolescent Rheumatology Etanercept Cohort Study. Rheumatology 2015;54:1279-85.

7. Wang SJ, Yang YH, Lin YT, Yang CM, Chiang BL. Attained adult height in juvenile rheumatoid arthritis with or without corticosteroid treatment. Clin Rheumatol 2002;21:363-8.

8. Simon D, Lucidarme N, Prieur AM, Ruiz JC, Czernichow P. Treatment of growth failure in juvenile chronic arthritis. Hormone Res 2002;58 Suppl 1:28-32.

9. Polito C, Strano CG, Olivieri AN, Alessio M, Iammarrone CS, Todisco N, et al. Growth retardation in non-steroid treated juvenile rheumatoid arthritis. Scand J Rheumatol 1997;26:99-103.

10. Gaspari S, Marcovecchio ML, Breda L, Chiarelli F. Growth in juvenile idiopathic arthritis: the role of inflammation. Clin Exp Rheumatol 2011;29:104-10.

11. Ansell BM, Bywaters EG. Growth in Still's disease. Ann Rheum Dis 1956;15:295-319.

12. Saha MT, Verronen P, Laippala P, Lenko HL. Growth of prepubertal children with juvenile chronic arthritis. Acta Paediatr 1999; 88:724-8.

13. Wong SC, Dobie R, Altowati MA, Werther GA, Farquharson C, Ahmed SF. Growth and the growth hormone-insulin like growth factor 1 axis in children with chronic inflammation: current evidence, gaps in knowledge, and future directions. Endocr Rev 2016;37:62-110.
14. De Benedetti F, Alonzi T, Moretta A, Lazzaro D, Costa P, Poli V, et al. Interleukin 6 causes growth impairment in transgenic mice through a decrease in insulin-like growth factor-I. A model for stunted growth in children with chronic inflammation. J Clin Invest 1997;99:643-50.

15. De Benedetti F, Rucci N, Del Fattore A, Peruzzi B, Paro R, Longo $\mathrm{M}$, et al. Impaired skeletal development in interleukin-6-transgenic mice: a model for the impact of chronic inflammation on the growing skeletal system. Arthritis Rheum 2006;54:3551-63.

16. De Benedetti F, Robbioni P, Massa M, Viola S, Albani S, Martini A Serum interleukin-6 levels and joint involvement in polyarticular and pauciarticular juvenile chronic arthritis. Clin Exp Rheumatol 1992;10:493-8.

17. De Benedetti F, Massa M, Robbioni P, Ravelli A, Burgio GR, Martini A. Correlation of serum interleukin-6 levels with joint involvement and thrombocytosis in systemic juvenile rheumatoid arthritis. Arthritis Rheum 1991;34:1158-63.

18. De Benedetti F, Brunner HI, Ruperto N, Kenwright A, Wright S, Calvo I, et al. Randomized trial of tocilizumab in systemic juvenile idiopathic arthritis. N Engl J Med 2012;367:2385-95.

19. Brunner HI, Ruperto N, Zuber Z, Keane C, Harari O, Kenwright A, et al; Paediatric Rheumatology International Trials Organisation PRINTO; Pediatric Rheumatology Collaborative Study Group (PRCSG). Efficacy and safety of tocilizumab in patients with polyarticular-course juvenile idiopathic arthritis: results from a phase 3, randomised, double-blind withdrawal trial. Ann Rheum Dis 2015;74:1110-7.

20. Ruperto N, Martini A. Networking in paediatrics: the example of the Paediatric Rheumatology International Trials Organisation (PRINTO). Arch Dis Child 2011;96:596-601.

21. Petty RE, Southwood TR, Manners P, Baum J, Glass DN, Goldenberg J, et al; International League of Associations for Rheumatology. International League of Associations for Rheumatology classification of juvenile idiopathic arthritis: second revision, Edmonton, 2001. J Rheumatol 2004;31:390-2.

22. World Health Organization. Child growth standards. WHO Anthro (version 3.2.2, January 2011) and macros. [Internet. Accessed April 18, 2018.] Available from: www.who.int/childgrowth/software/en

23. Tanner JM. Normal growth and techniques of growth assessment. Clin Endocrinol Metab 1986;15:411-51.

24. Brunner HI, Ruperto N, Zuber Z, Cuttica RJ, Xavier R, Calvo I, et al. Efficacy and safety of tocilizumab in patients with polyarticular-course juvenile idiopathic arthritis: 2-year data from Cherish [abstract]. Arthritis Rheum 2013;65:S335.

25. Simon D, Fernando C, Czernichow P, Prieur AM. Linear growth and final height in patients with systemic juvenile idiopathic arthritis treated with longterm glucocorticoids. J Rheumatol 2002; 29:1296-300. 